\title{
Insight of Mechanism and Signaling Pathway in Pathogenesis of Diabetic Neuropathy: A Review
}

\author{
Norhamidar A.H. ${ }^{a}$, Norsuhana O. ${ }^{b}$, Che Aishah Nazariah I. ${ }^{b}$, Idris L. ${ }^{a}$ \\ ${ }^{a}$ School of Health Sciences, Health Campus, Universiti Sains Malaysia \\ ${ }^{b}$ School of Medical Sciences, Health Campus, Universiti Sains Malaysia
}

\begin{abstract}
Keywords
Diabetic neuropathy, hyperglycaemia, signaling pathways, glucotoxicity, vascular dysfunction, oxidative stress.

Corresponding Author

Dr. Idris Long

School of Health Sciences, Health

Campus, Universiti Sains Malaysia, 16150

Kubang Kerian, Kelantan, Malaysia.

Tel No: +609-7677778

E-mail: idriskk@usm.my

Received: 12 July 2021; Accepted: 22 August 2021

Doi: https://doi.org/10.31436/imjm.v20i4
\end{abstract}

\begin{abstract}
Diabetic neuropathy (DN) is a common chronic microvascular complication of diabetes mellitus. The features of DN include allodynia, hyperalgesia, abnormal or loss of sensation of nerve fibers. The clinical features will contribute to poor quality of life, disrupt sleep, lead to depression, and increases mortality. Current drug treatments have been shown to alleviate the symptoms of DN but failed to treat the underlying causes of DN. Therefore, a better understanding of the molecular mechanisms underlying the development and progression of $\mathrm{DN}$ is needed for early diagnosis and intervention and understanding the failure of existing treatments. Identification of potential mechanisms is critical for better prediction of progression and for designing preventive therapies. DN's exact pathogenesis is incomplete, although it is understood that its multifunctional dysfunction involving many signaling pathways. This review summarised the common deterioration of signaling pathways and mechanisms involved in DN pathogenesis.
\end{abstract}

\section{INTRODUCTION}

Diabetes Mellitus (DM) is a significant worldwide public health concern. In 2017, 425 million people worldwide had diabetes, according to the International Diabetes Federation, and this figure is projected to rise to 629 million by $2045 .{ }^{1}$ A dramatic spike in the prevalence of diabetes has also been seen in Asia. According to the International Diabetes Federation, 82 million people in Southeast Asia have diabetes. According to Malaysia's National Health and Morbidity Survey (NHMS), the prevalence of a known or established diagnosis of diabetes during the 2019 NHMS was $9.4 \%$, whilst in 2015 it was $8.3 \%$. Hence in 2019, there were almost 2 million $(1,999,450)$ adult individuals with known diabetes in Malaysia. ${ }^{2}$

Diabetic neuropathy (DN) is the common chronic microvascular complication symptom of diabetes, affecting about half of all diabetic patients. It is projected that 50 percent of patients develop symptoms of nerve injury after having $\mathrm{DN}$ for over 25 years. ${ }^{3} \mathrm{DN}$ can be characterised as symmetrical, length-dependent sensorimotor polyneuropathy attributable to metabolic and microvessel alterations due to chronic hyperglycaemia and hyperlipidaemia.

DN has two principal clinical manifestations known as painless and painful DN. Painless DN is characterised by a lack of sensitivity to pain, which can cause foot ulcers, while painful DN is characterised by neuropathic pain that can be highly distressing. Numbness, burning, "electric-shock type" pain, aching, scratching, and cold pain are all signs of DN. These signs lead to diminished 
daily life, higher unemployment rates, sleep disruption, stress and mental health problems, physical comorbidities, and even amputation. ${ }^{4}$

\section{Pathogenesis of DN}

The exact pathogenesis of DN is incomplete, although it is understood that the multifunctional dysfunction of DN results from many impaired signaling pathways. The common deterioration of signaling pathways and mechanisms involved in the DN pathogenesis are summarised in this review.

\section{Glucotoxicity}

Prolonged hyperglycaemia in diabetes can induce glucotoxicity which impairs numerous pathways in the biological metabolome.

\section{Polyol pathway}

In this pathway, glucose is converted to sorbitol by the enzyme aldose reductase. Then sorbitol is further metabolised to fructose by the sorbitol dehydrogenase enzyme. The conversion of glucose into sorbitol by aldose reductase uses nicotinic acid adenine dinucleotide phosphate (NADPH), while to convert fructose to sorbitol, nicotinic acid adenosine dinucleotide (NAD) is used as a cofactor. 5 Through this pathway, the cell redox state is altered by regulating the NADPH/ $\mathrm{NADP}^{+}$and $\mathrm{NADH} / \mathrm{NAD}^{+}$ratios. The $\mathrm{NADPH}$ is the cofactor that is necessary to produce glutathione (GSH), an antioxidant scavenger for reactive oxygen species (ROS). The overconsumption of NADPH to convert glucose to sorbitol may cause depletion of GSH production and therefore, exacerbates the effect of intracellular oxidative stress. The aldose reductase activity also further depletes cellular stores of NADPH, which are needed for the generation of nitric oxide (NO), the vasodilator agent of the blood vessel. ${ }^{6}$ The increase of sorbitol production also can create an osmotic imbalance in the cell and this situation is compensating by efflux of myo-inositol and taurine. The efflux of myo-inositol, which is an essential component of the $\mathrm{Na}^{+} / \mathrm{K}^{+}$-ATPase pump makes myoinositol concentration in the cell is reduced. All of these processes result in the generation of cytoplasmic ROS

\section{Production of Advanced glycation end product (AGE)}

Hyperglycaemia in diabetes may also trigger the production of advanced glycation end product (AGE). The non-enzymatic reaction between aldehyde groups of reducing sugars with protein amino groups, lipids, or nucleic acids gives rise to the formation of reactive dicarbonyl intermediate (glyoxal, methylglyoxal) by auto -oxidation and a variety of AGEs. ${ }^{5}$ As a consequence of this glucose auto-oxidation and formation of AGEs, hydroxyl radicals and hydrogen peroxide are produced. ${ }^{8}$ The interaction of AGEs with the AGE receptor (RAGE) can activate the downstream signaling cascade by nuclear factor kappa-light-chain-enhancer of activated $\mathrm{B}$ cells $(\mathrm{NF}-\mathrm{kB})$ that can induce inflammation in the cells. Besides the production of AGEs, lipid metabolism is also affected during diabetes. Diabetes may increase the development of lipid peroxidation as a result of the enhanced oxidative stress production and increase the production of lipoxidation end products (ALEs) that are chemically similar to AGEs.?

\section{Poly(ADP-ribose) polymerase (PARP)}

In the normal condition, the PARP enzyme is associated with DNA repairing and apoptosis mechanism. In DM, hyperglycaemia leads to the generation of reactive oxygen and nitrogen species, which initiate single-strand DNA breaks and activate the PARP enzyme. ${ }^{5}$ However, when the PARP enzyme is highly activated, it causes depletion of intracellular $\mathrm{NAD}+/ \mathrm{NADPH}$ and generation of ADP ribosylation of glyceraldehydes 3-phosphate dehydrogenase (GAPDH). This process causes energy failure, alteration of transcriptional regulation and gene expression that upregulates the release of proinflammatory mediators, and a shift of the glycolytic flux into several pathways associated with nerve blood vessel damage. 10

\section{Hexosamide pathway}

In the hexosamine pathway, fructose-6-phosphate is diverted from glycolysis and is transformed into glucosamine-6-phosphate. Glucosamine-6-phosphate amidotransferase enzyme further transforms the 
glucosamine-6-phosphate into uridine phosphate-Nacetyl glucosamine (UDPGlcNAc). The N-acetyl glucosamine (GlcNAc) can induce $\beta$-cell function deficit due to the production of oxidative stress. ${ }^{11}$ The higher level of glucosamine-6-phosphate amidotransferase enzyme due to hyperglycemia, increased hydrogen peroxide levels that can inhibit insulin production, glucose transporter 2, and glucokinase genes expression. ${ }^{12}$ Increased activity of hexosamine pathway also induces changes in gene transcription factor Sp1 that controls gene expression of transforming growth factor $\beta 1$ (TGF- $\beta 1$ ) and plasminogen activator inhibitor-1 (PAI-1). The upregulation of TGF- $\beta 1$ activated collagen matrix production is associated with endothelial fibrosis and inhibition of mesangial cell proliferation. Meanwhile, the increased PAI-1 may enhance vascular smooth muscle cells that are responsible for atherosclerosis formation. ${ }^{13}$

\section{Upregulation of Oxidative stress}

In the pathogenesis of DN, oxidative stress is also known to involve in modifying certain signalling pathways and tissue structures. The occurrence of prolonged hyperglycaemia leads to the auto-oxidation of glucose in several cells and tissues as a result of failure to detoxify the endogenous release of free radicals. ${ }^{14}$ This mechanism results in the activation of the polyol pathway that further converts glucose to fructose with the further action of sorbitol dehydrogenase. These biological systems implicate the formation of nicotinamide adenine dinucleotide phosphate (NADPH) directly that in turn to decreased production of glutathione (GSH), a primary endogenous antioxidant. These overall modifications may end up in the hyper release of oxidative stress and failure of nerve conduction. ${ }^{14}$ In fact, the increased level of oxidative stress markers in the early phenomenon detected in the occurrence of insulin resistance, modification of aldose-reductase pathway, and inflammation. 14,15

Hydrogen peroxide $\left(\mathrm{H}_{2} \mathrm{O}_{2}\right)$, nitric oxide, and superoxide anion $\left(\mathrm{O}_{2}-\right)$ are the essential free radicals produced endogenously during normal physiology. During metabolic dysfunction, the increased level of oxidative stress may lead to cellular damage. The increased production of $\mathrm{O}_{2}$ - in $\beta$-cell may induce stresssignalling pathways to activate downstream effectors including NF-kB. These mechanisms may cause the dysfunction and apoptosis of $\beta$-cell and further implicates insulin secretion. ${ }^{16}$ Other than that, $\mathrm{H}_{2} \mathrm{O}_{2}$, a potent oxidising agent is yielded from the superoxide dismutase (SOD)-catalysed dismutation of $\mathrm{O}_{2}{ }^{-}$. The released hydroxyl radicals may cause serious biological effects such as impaired vasodilation that may damage endothelial cells and tissue hypoxia. ${ }^{17}$ Meanwhile, the increased lipid peroxidation may also occur due to the activity of ROS in attacking plasma lipid, endoplasmic reticulum, and mitochondria. ${ }^{17}$

\section{Protein Kinase C (PKC) pathway}

Prolonged hyperglycaemia and increased glycolysis lead to the accumulation of dihydroxy-acetone phosphate that is further converted to diacylglycerol (DAG). The production of DAG may activate the neuronal PKC. $\mathrm{PKC}$ is a serine/threonine-protein kinase family involved in many cellular functions and signaling transduction pathways for cell proliferation, differentiation, and apoptosis. ${ }^{5}$ There are fifteen isozymes of PKC family that can be divided into conventional $(\alpha, \beta I, \beta I I$, and $\gamma)$, novel $(\delta, \varepsilon, \eta$, and $\theta)$ and atypical (M $\zeta$ and $\iota / \lambda$ ) that require $\mathrm{DAG}$ for activation.

The activation of PKC may decrease the $\mathrm{Na}^{+} / \mathrm{K}^{+}$ATPase activity in smooth muscle cells and upregulate vascular endothelial growth factor (VEGF), TGF- $\beta 1$, PAI-1 and NF-kB that play a major role in various diabetic complications including retinopathy, nephropathy, and cardiovascular diseases. 5, 18, 19

Furthermore, PKC activation may also alter vasoconstriction and capillary permeability and implicates hypoxia, angiogenesis, thickening of the basement membrane and endothelial proliferation. ${ }^{20} \mathrm{It}$ is also reported that the activation of PKC- $\beta$ activity may also be a source of excess production of free radicals, leading to the increased generation of superoxide and depletion of NO in vascular endothelial cells. 


\section{Activation of inflammatory pathway}

Hyperglycaemia in diabetes also triggers many inflammation signaling pathways that excretes various mediators that worsen the condition and contributing to the development of DN.

\section{Mitogen-activated protein kinases (MAPKs) pathway}

The MAPKs are intracellular components that play a role in signaling from cell membrane receptors to the nucleus. MAPK families include p38, extracellular signal -related kinase (ERK), and c-Jun N-terminal kinase (JNK). ${ }^{21}$ According to Xia et al. (1995) ${ }^{22}$, ERK is responsible for brain survival, whereas JNK and p38 are responsible for neural death. However, ERK is engaged in and plays a crucial part in the pathogenesis of neuropathic pain. ${ }^{23}$ Upregulation of ERK, p38, and JNK has been identified in the spinal ganglion neuron of STZ-induced diabetic rats. ${ }^{24}$ In diabetic rats and mice, inhibiting p38 MAPK improves nerve conduction velocity ${ }^{25}$, produces antinociceptive effect ${ }^{26}$, corrects erectile dysfunction ${ }^{27}$, improves vascular endotheliummediated vasodilation, and decreases endothelial activation. ${ }^{28}$ The elevated levels of p38 and JNK have been reported in the sural nerves of type 1 and type 2 diabetes individuals. ${ }^{29,30} \mathrm{JNK}$ activity has also been connected to neurofilament phosphorylation in the dorsal root ganglion (DRG) of diabetic rats. ${ }^{31}$ It has been proposed that inhibiting the persistent JNK activation in DRG neurons may enhance neuronal regeneration in diabetic rats.

\section{Nuclear Factor kappa-light-chain-enhancer B (NF-KB) pathway}

The NF- $\mathrm{KB}$ is a transcriptional factor that plays a critical role in mediating immune and inflammatory responses and apoptosis. The NF- $\mathrm{KB}$ is activated by other pro-inflammatory insults like TNF- $\alpha$ and IL-1 $\beta$ and oxidative stress markers following the constant hyperglycaemic condition. These mechanisms are occurred via the activation of Toll-like receptors (TLRs) by these inflammatory cytokines in the canonical pathway. ${ }^{15,32}$ These mechanisms further result in the
I $\mathrm{KB}$ degradation as a result of the phosphorylation of the multi-subunit IKB kinase (IKK) complex. Once activated, the IKK phosphorylates IKBa at two Nterminal serines that stimulates the degradation of IKBa in the proteasome. As a result, the canonical NF- $x \mathrm{~B}$ members such as p50/ReIA and p50/c-Rel dimers translocate into the nucleus 33 to induce the NF- $\mathrm{KB}$ dependent genes such as pro-IL-1 $\beta$, pro-IL-18, and Nod -like receptor protein 3 (NLRP3). It has been shown that the NLRP3 inflammasome is increased in the sciatic nerve of STZ-induced DN rats. ${ }^{34}$ Other than that, the increased activation of NF- $\mathrm{KB}$ may also stimulate the productions of other pro-inflammatory and immune cells such as T-cells that further damages the endothelial cells. ${ }^{35} \mathrm{Li}$ et al (2016) ${ }^{36}$ demonstrated the co-localisation of NF- $\kappa B$ p65 with NADPH oxygenase-2 (NOX2) and NADPH oxygenase-4 (NOX4) in the aorta of DN rat. This study suggests the possible action of NF- $\mathrm{\kappa B}$ in stimulating the NOXs activation and ROS overproduction following the persistent increase in blood glucose level. ${ }^{36}$ Other than that, the NF- $\mathrm{BB}$ transcription can also cause the increased level of heme oxygenase-1 (HO-1), a stress protein marker available in the astrocyte culture of the rat's brain. ${ }^{37}$

\section{Glial cells activation}

An increasing amount of research suggests that nonneuronal cells such as microglia and astrocytes become activated during the pathogenesis of $\mathrm{DN}$ in a hyperglycaemic environment. ${ }^{38}$ Previous research has shown that glial-neuron crosstalk causes pathological pain in DN rat models, including allodynia and hyperalgesia, via the release of numerous inflammatory mediators. According to Barragán-Iglesias et al (2018) 39, the DN neonate Wistar rat model has increased glial fibrillary acidic protein (GFAP) and OX-42 immunoreactivity (remarks astrocyte and microglia expressions, respectively) with a marked increase in the hypertrophied and amoeboid-like structure of microglia in the spinal cord as well as increased satellite glial cells (SGCs) in the dorsal root ganglion (DRG). The increased expression of these glial cells, both peripherally and centrally, has been demonstrated to be strongly related to the development of tactile allodynia in this 
neonatal rat model, indicating their importance in the pathogenesis of DN. In another work, Ismail et al (2020) ${ }^{40}$, discovered an early increase in the expression of OX-42 positive cells in the spinal cord of a painful DN rat model, which might explain the heightened formalin-induced pain behaviour responses in this rat model. The study also found the increased immunoreactivity of spinal brain-derived neurotrophic factor (BDNF) and downstream regulatory element antagonist modulator (DREAM), which are likely linked to the increased microglia-neuron crosstalk because DREAM is transcribed in neurons in response to the increased calcium ion $\left(\mathrm{Ca}^{2+}\right)$ influx from the activated $\mathrm{N}$ -Methyl-D-aspartate receptors (NMDARs). Minocycline appears to restore the allodynic and hyperalgesic state in painful DN rats by attenuating the microglia activation in conjunction with BDNF and DREAM signaling proteins. 41

\section{Vascular impairment}

Prolonged hyperglycaemic conditions in diabetes can affect the functions and structures of the peripheral and central nervous systems. It causes dysfunction of nerve blood flow and facilitates nerve hypoxia. ${ }^{42}$ Many animal studies, such as the rats treated with streptozotocin (STZ), showed diminished nerve blood supply within a few days of diabetes induction. ${ }^{43}$ It is possible to apply this procedure to other animal models. Diabetes can induce endoneurial hypoxia that impairs nerve activity and triggers neurodegenerative processes by decreasing nerve blood flow. This phenomenon exists in the peripheral nerve and other central nervous system components, such as the dorsal root ganglia (DRG) and also the hippocampus brain structure. ${ }^{44}$

In diabetes, nerve tissue perfusion deficiency is thought to be due to a process known as vasa nervorum endotheliopathy. The alteration in diabetes metabolic demand induces an imbalance in the production and effects of major blood vessel vasodilators such as nitric oxide, hyperpolarizing factor derived from endothelium (EDHF), and prostacyclin.

It decreases the effects of vasodilation in the bloodstream and potentiates high diabetes vasoconstriction agents such as angiotensin II and endothelin 1.45 In nerve electrophysiology, multiple experiments using various vasodilator agents such as angiotensin-converting enzyme inhibitors, calcium channel blockers, endothelin-1 receptor antagonists, and alpha1 adrenoceptor blockers have demonstrated progress, correlating with the degree of correction of diabetic endoneurial perfusion deficiency. ${ }^{44,45}$

Hyperglycaemia due to diabetes has also been reported to cause structural changes in nerve microvasculature, including thickening of the basement membrane, degeneration of pericytes, and hyperplasia of endothelial cells. ${ }^{46}$

The probability of structural changes are extracted from the maladaptive reaction of endoneurial hypoxia that induces decreased diffusion capacity, which further exacerbates hypoxia. ${ }^{47}$ By controlling arterio-venous shunting in the epineural and perineurial feeding arteriole, unmyelinated nerve fibers engage in managing endoneurial blood flow. However, due to injury to this fiber in the early neuropathic phase, the innervation between an arteriole and a venous was shunt, and the state of hypoxia became worse. 48 In addition to microvascular risk factors, DN may also be associated with risk factors for macrovascular disorders such as hypertension, tobacco smoking, dyslipidemia, microalbuminuria, and body mass index rises (BMI). ${ }^{44,45}$

\section{Conclusion and future prospect}

$\mathrm{DN}$ is a frequent consequence of diabetes that lowers the quality of life and increases mortality. So far, current therapies have just relieved symptoms rather than elucidating the fundamental process in developing DN. This review has shown a complex etiology of interconnected advanced glycation/lipoxidation, PARP activity, oxidative/nitrosative stress, activation of proinflammatory mediators, and neurovascular networks in the pathogenesis of DN as illustrated in Figure 1. There can have adverse effects on neurons directly, as well as indirectly through decreased nerve vascular supply. There are potential treatment targets specific to all of these signaling pathways that should be explored in clinical trials for maximum treatment efficacy for $\mathrm{DN}$. 


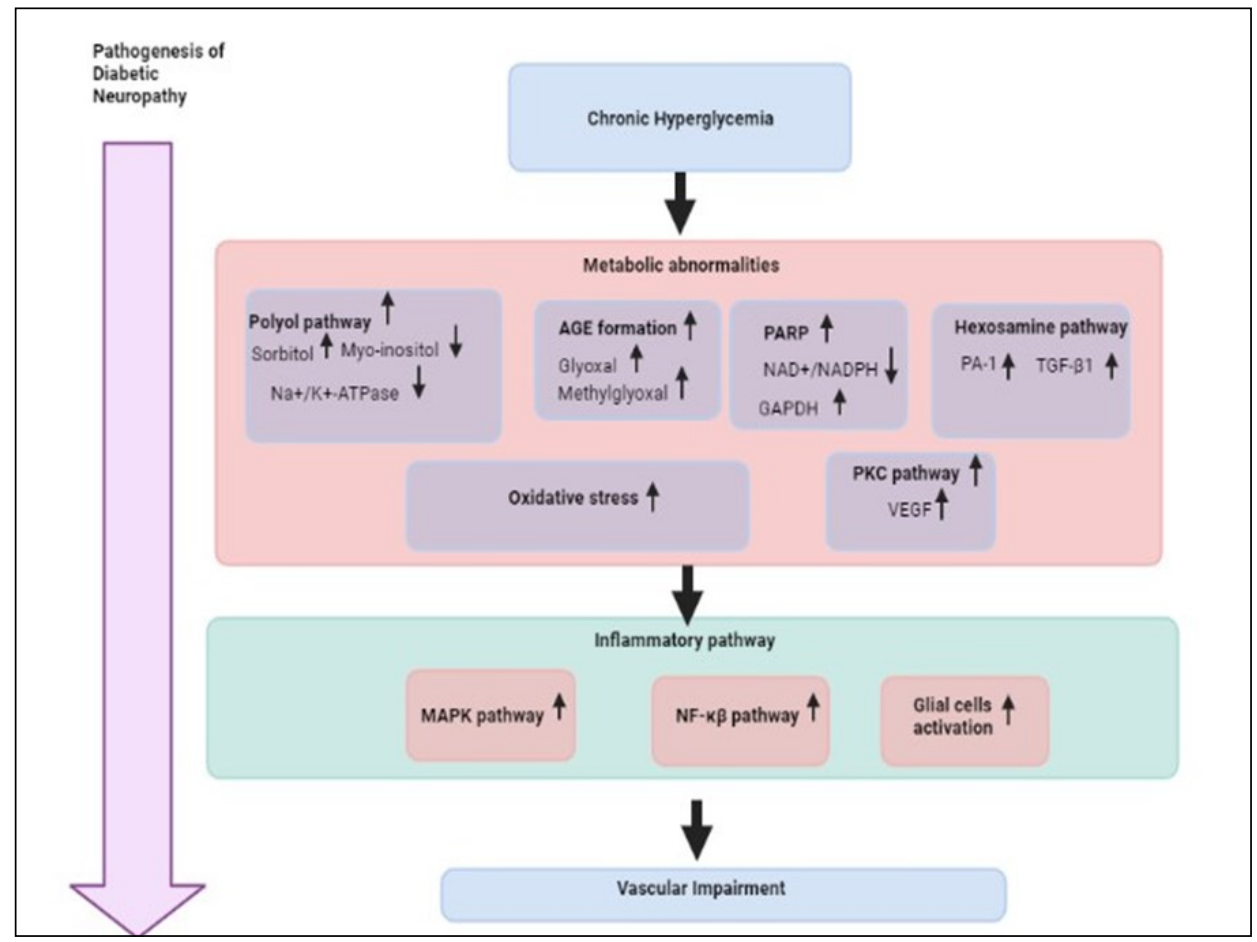

Figure 1: Sequence of events that lead to diabetic neuropathy. The metabolic problems caused by persistent hyperglycemia are frequently complex and mutually reinforcing of many pathways. They cause the neuroinflammation pathway to be activated, resulting in structural and functional disruption of the blood vessel. AGE, advanced glycation end product; PARP, Poly(ADP-ribose) polymerase; PKC, protein kinase C; NAD, nicotinic acid adenosine dinucleotide; NADPH, nicotinic acid adenine dinucleotide phosphate; GAPDH, glyceraldehydes 3-phosphate dehydrogenase; TGF- $\beta$, transforming growth factor $\beta 1$ PA-1, plasminogen activator inhibitor-1; VEGF, vascular endothelial growth factor; MAPK, Mitogen-activated protein kinases; NF-KB, Nuclear Factor kappa-light-chainenhancer B. (Adapted from Feldman et al., 2017) ${ }^{3}$

\section{DECLARATION OF CONFLICTING INTERESTS}

The author(s) declared no potential conflicts of interest concerning the research, authorship, and/or publication of this article.

\section{ACKNOWLEDGMENT}

This study was supported by Fundamental Research Grant Scheme (FRGS) Ministry of Higher Education Malaysia (FRGS/1/2020/SKK0/USM/03/5).

\section{REFERENCES}

1. Nam Han Cho et al. Eighth edition 2017. IDF Diabetes Atlas, 8th edition. 2017. 1-150. Available from: https://www.idf.org/aboutdiabetes/type-2diabetes.html
2. National health and Morbidity survey 2019.

Non-communicable diseases, risk factors and other health problem; volume II, Institute for public health, Ministry of Health Malaysia.

3. Feldman EL, Nave KA, Jensen TS, Bennett DLH. New Horizons in Diabetic Neuropathy: Mechanisms, Bioenergetics, and Pain. Neuron. 2017;93(6):1296-313. Available from: http:// dx.doi.org/10.1016/j.neuron.2017.02.005

4. Gylfadottir SS, Weeracharoenkul D, Andersen ST, Niruthisard S, Suwanwalaikorn S, Jensen TS. Painful and non-painful diabetic polyneuropathy: Clinical characteristics and diagnostic issues. J Diabetes Investig. 2019;10(5):1148-57.

5. Bhattacharjee N, Barma S, Konwar N, Dewanjee $S$, Manna P. Mechanistic insight of diabetic nephropathy and its pharmacotherapeutic targets: An update. Eur J Pharmacol. 2016;791:8-24. Available from: http://dx.doi.org/10.1016/ j.ejphar.2016.08.022 
6. Lee AYW, Chung SSM. Contributions of polyol pathway to oxidative stress in diabetic cataract. FASEB J. 1999;13(1):23-30.

7. Oates P. Aldose Reductase, Still a Compelling Target for Diabetic Neuropathy. Curr Drug Targets. 2008;9(1):14-36.

8. Vincent AM, Perrone L, Sullivan KA, Backus C, Sastry AM, Lastoskie C, et al. Receptor for advanced glycation end products activation injures primary sensory neurons via oxidative stress. Endocrinology. 2007;148(2):548-58.

9. Zochodne DW. Mechanisms of diabetic neuron damage: Molecular pathways [Internet]. 1st ed. Vol. 126, Handbook of Clinical Neurology. Elsevier B.V.; 2014. 379-399 p. Available from: http://dx.doi.org/10.1016/B978-0-444-534804.00028-X

10. Homburg S, Visochek L, Moran N, Dantzer F, Priel E, Asculai E, et al. A fast signal-induced activation of poly(ADP-ribose) polymerase: $\mathrm{A}$ novel downstream target of phospholipase C. J Cell Biol. 2000;150(2):293-307.

11. Brownlee M. Biology of Diabetic Complications. Nature. 2001;414(December):813-20.

12. Kaneto H, Xu G, Song KH, Suzuma K, BonnerWeir S, Sharma A, et al. Activation of the Hexosamine Pathway Leads to Deterioration of Pancreatic $\beta$-Cell Function through the Induction of Oxidative Stress. J Biol Chem. 2001;276

(33):31099-104. Available from: http:// dx.doi.org/10.1074/jbc.M104115200

13. Sayeski PP, Kudlow JE. Glucose metabolism to glucosamine is necessary for glucose stimulation of transforming growth factor- $\alpha$ gene transcription. J Biol Chem. 1996;271(25):1523743. Available from: http://dx.doi.org/10.1074/ jbc.271.25.15237

14. Adki KM, Kulkarni YA. Biomarkers in diabetic neuropathy. Arch Physiol Biochem. 2020 Nov 13;1-16. Available from: https:// doi.org/10.1080/13813455.2020.1837183

15. Yu Z-W. Role of nuclear factor (erythroid-derived 2)-like 2 in metabolic homeostasis and insulin action: A novel opportunity for diabetes treatment? World J Diabetes. 2012;3(1):19.

16. Oyenihi AB, Ayeleso AO, Mukwevho E, Masola B. Antioxidant strategies in the management of diabetic neuropathy. Biomed Res Int. 2015;2015.
17. Vincent AM, Russell JW, Low P, Feldman EL. Oxidative stress in the pathogenesis of diabetic neuropathy. Endocr Rev. 2004;25(4):612-28.

18. Veves A, King GL. Can VEGF reverse diabetic neuropathy in human subjects? J Clin Invest. 2001;107(10):1215-8.

19. Khanra R, Dewanjee S, K Dua T, Sahu R, Gangopadhyay M, DeFeo V, et al. Abroma augusta L. (Malvaceae) leaf extract attenuates diabetes induced nephropathy and cardiomyopathy via inhibition of oxidative stress and inflammatory response. J Transl Med. 2015;13(1):1-14.

20. Edwards AS, Faux MC, Scott JD, Newton AC. Carboxyl-terminal phosphorylation regulates the function and subcellular localization of protein kinase C $\beta$ II. J Biol Chem. 1999;274(10):6461-8. Available from: http://dx.doi.org/10.1074/ jbc.274.10.6461

21. Das S, Joardar S, Manna P, Dua TK, Bhattacharjee N, Khanra R, et al. Carnosic Acid, a Natural Diterpene, AttenuatesArsenic-Induced Hepatotoxicity.2018;2018.

22. Xia P, Kramer RM, King GL. Identification of the mechanism for the inhibition of $\mathrm{Na}+, \mathrm{K}+$ adenosine triphosphatase by hyperglycemia involving activation of protein kinase $\mathrm{C}$ and cytosolic phospholipase A2. J Clin Invest. 1995;96(2):733-40.

23. Peng G, Han M, Du Y, Lin A, Yu L, Zhang Y, et al. SIP30 is regulated by ERK in peripheral nerve injury-induced neuropathic pain. J Biol Chem. 2009;284(44):30138-47. Available from: http:// dx.doi.org/10.1074/jbc.M109.036756

24. Daulhac L, Mallet C, Courteix C, Etienne M, Duroux E, Privat AM, et al. Diabetes-induced mechanical hyperalgesia involves spinal mitogenactivated protein kinase activation in neurons and microglia via N-methyl-D-aspartate- dependent mechanisms. Mol Pharmacol. 2006;70(4):124654.

25. Price SA, Agthong S, Middlemas AB, Tomlinson DR. Mitogen-activated protein kinase p38 mediates reduced nerve conduction in experimental diabetic neuropathy: Interactions with aldose reductase. Diabetes. 2004;53(7):18516.

26. Sweitzer SM, Medicherla S, Almirez R, Dugar S, 
Chakravarty S, Shumilla JA, et al. Antinociceptive action of a $\mathrm{p} 38 \alpha \mathrm{MAPK}$ inhibitor, SD-282, in a diabetic neuropathy model. Pain. 2004;109(3):409 -19 .

27. Toque HA, Romero MJ, Tostes RC, Shatanawi A, Chandra S, Carneiro ZN, et al. NIH Public Access. 2011;7(12):3857-67.

28. Yu Y, Rajapakse AG, Montani JP, Yang Z, Ming $\mathrm{XF}$. P38 mitogen-activated protein kinase is involved in arginase-II-mediated eNOSUncoupling in Obesity. Cardiovasc Diabetol. 2014;13(1):1-10.

29. Purves T, Middlemas A, Agthong S, Jude EB, Boulton AJM, Fernyhough, et al. A role for mitogen-activated protein kinases in the etiology of diabetic neuropathy. FASEB J. 2001;15 (13):2508-14.

30. Tomlinson DR, Gardiner NJ. Glucose neurotoxicity. Nat Rev Neurosci. 2008;9(1):36-45.

31. Fernyhough P, Gallagher A, Averill SA, Priestley J V., Hounsom L, Patel J, et al. Aberrant neurofilament phosphorylation in sensory neurons of rats with diabetic neuropathy. Diabetes. 1999;48(4):881-9.

32. Lawrence T. The nuclear factor NF-kappaB pathway in inflammation. Cold Spring Harb Perspect Biol. 2009;1(6):1-11.

33. Liu T, Zhang L, Joo D, Sun SC. NF-xB signaling in inflammation. Signal Transduct Target Ther. 2017;2(March).

34. Sun Q, Wang C, Yan B, Shi X, Shi Y, Qu L, et al. Jinmaitong ameliorates diabetic peripheral neuropathy through suppressing TXNIP/NLRP3 inflammasome activation in the streptozotocininduced diabetic rat model. Diabetes, Metab Syndr Obes Targets Ther. 2019;12:2145-55.

35. Suryavanshi S V., Kulkarni YA. NF-xß: A potential target in the management of vascular complications of diabetes. Front Pharmacol. 2017;8(NOV):1-12.

36. Li W, Zhao W, Wu Q, Lu Y, Shi J, Chen X. Puerarin Improves Diabetic Aorta Injury by Inhibiting NADPH Oxidase-Derived Oxidative Stress in STZ-Induced Diabetic Rats. J Diabetes Res. 2016;2016.

37. Yang H, Sloan G, Ye Y, Wang S, Duan B, Tesfaye $\mathrm{S}$, et al. New Perspective in Diabetic Neuropathy:
From the Periphery to the Brain, a Call for Early Detection, and Precision Medicine. Front Endocrinol (Lausanne). 2020;10(January):1-13.

38. Wang D, Couture R, Hong Y. Activated microglia in the spinal cord underlies diabetic neuropathic pain. Eur J Pharmacol. 2014;728(1):59-66. Available from: http://dx.doi.org/10.1016/ j.ejphar.2014.01.057

39. Barragán-Iglesias P, Oidor-Chan VH, LoezaAlcocer E, et al. Evaluation of the neonatal streptozotocin model of diabetes in rats: evidence for a model of neuropathic pain. Pharmacol Rep. 2018 70: 294-303.

40. Ismail CAN, Suppian R, Ab Aziz CB, Long I. Expressions of spinal microglia activation, BDNF, and DREAM proteins correlated with formalininduced nociceptive responses in painful and painless diabetic neuropathy rats. Neuropeptides. 2020;79(June 2019):102003. Available from: https://doi.org/10.1016/j.npep.2019.102003

41. Ismail CAN, Suppian R, Aziz CBA, Long I. Minocycline attenuates the development of diabetic neuropathy by modulating DREAM and BDNF protein expression in rat spinal cord. J Diabetes Metab Disord. 2019;18, 181-190.

42. Tesfaye S, Chaturvedi N, Eaton SEM, Ward JD, Manes C, Ionescu-Tirgoviste C, et al. Vascular Risk Factors and Diabetic Neuropathy. N Engl J Med. 2005;352(4):341-50.

43. Coppey LJ, Gellett JS, Davidson EP, Dunlap JA, Lund DD, Yorek MA. Effect of Antioxidant Treatment of Streptozotocin-Induced Diabetic Rats on Endoneurial Blood Flow, Motor Nerve Conduction Velocity, and Vascular Reactivity of Epineurial Arterioles of the Sciatic Nerve.

Diabetes. 2001;50(8):1927-37.

44. Sytze Van Dam P, Cotter MA, Bravenboer B, Cameron NE. Pathogenesis of diabetic neuropathy: Focus on neurovascular mechanisms. Eur J Pharmacol. 2013;719(1-3):180-6. Available from: http://dx.doi.org/10.1016/ j.ejphar.2013.07.017

45. Cameron NE, Eaton SEM, Cotter MA, Tesfaye S. Vascular factors and metabolic interactions in the pathogenesis of diabetic neuropathy. Diabetologia. 2001;44(11):1973-88.

46. Shimizu F, Sano Y, Haruki H, Kanda T. Advanced 
glycation end-products induce basement

membrane hypertrophy in endoneurial

microvessels and disrupt the blood - Nerve barrier

by stimulating the release of TGF- $\beta$ and vascular

endothelial growth factor (VEGF) by pericytes.

Diabetologia. 2011;54(6):1517-26.

47. Østergaard L, Finnerup NB, Terkelsen AJ, Olesen RA, Drasbek KR, Knudsen L, et al. The effects of capillary dysfunction on oxygen and glucose extraction in diabetic neuropathy. Diabetologia. 2015;58(4):666-77.

48. Tesfaye S, Harris N, Jakubowski JJ, Mody C, Wilson RM, Rennie IG, et al. Diabetologia. 1993;1266-74. 